Proceedings

\title{
Experimental Characterization of the Self-Healing Capacity of Cement Based Materials: An Overview ${ }^{\dagger}$
}

\author{
Liberato Ferrara 1,*, Estefania Cuenca Asensio 1, Francesco Lo Monte 1, Marta Roig Flores ${ }^{2}$, \\ Mercedes Sanchez Moreno ${ }^{3}$, Didier Snoeck ${ }^{4}$, Tim Van Mullem ${ }^{4}$ and Nele De Belie ${ }^{4}$ \\ 1 Department of Civil and Environmental Engineering, Politecnico di Milano, 20133 Milano, Italy; \\ estefania.cuenca@polimi.it (E.C.A.); francesco.lo@polimi.it (F.L.M.) \\ 2 CSIC-Eduardo Torroja Institute for Construction Science, 28033 Madrid, Spain; marta.roig@ietcc.csic.es \\ 3 Department of Inorganic Chemistry and Chemical Engineering, University of Cordoba, 14071 Cordoba, \\ Spain; msmoreno@uco.es \\ 4 Magnel Laboratory for Concrete Research, Faculty of Engineering and Architecture, Ghent University, \\ 9000 Ghent, Belgium; didier.snoeck@ugent.be (D.S.); Tim.VanMullem@ugent.be (T.V.M.); \\ nele.debelie@ugent.be (N.D.B.) \\ * Correspondence: liberato.ferrara@polimi.it; Tel.: +39-02-2399-4387 \\ + Presented at the 18th International Conference on Experimental Mechanics (ICEM18), Brussels, Belgium, \\ 1-5 July 2018.
}

Published: 11 June 2018

\begin{abstract}
This paper, prepared in the framework of the COST Action SARCOS WG2 activities (http://www.cost.eu/COST_Actions/ca/CA15202), provides an overview of the main concepts underlying the experimental characterization of the self-sealing capacity of cement-based materials. This effort intends to pave the way towards standardization of such experimental methods for a comparative assessment of the self-healing capacity of different cement-based materials and of the effectiveness of different techniques, in order to incorporate self-healing concepts and outcomes into durability-based predictive models and design approaches.
\end{abstract}

Keywords: self-healing; cement-based materials; durability and mechanical properties recovery

\section{Introduction}

The identification of material properties to be employed in structural analysis and design procedures is one of the main goals of experimental mechanics in the field of concrete and cementbased construction materials, together with the validation of the same design procedures. This issue has become of the foremost importance as long as the cement and construction industry has been providing, all along the last decades, a variety of advanced constituents, materials concepts, construction products, structural and construction elements whose peculiar features make them hardly classifiable into categories already covered by current test and product standards and design codes.

In the last decade a really giant leap forward has been made with reference to the concept and development of self-healing concrete and cement-based materials. Their implementation in the construction industry has been recognized as a pathway worth being travelled with the aim of improving the durability and long-term performance of structures made of and/or retrofitted with the aforementioned category of construction materials, able to extend their lifespan, also through the incorporation of new advanced functionalities to traditional materials. The variety of materials and techniques to either stimulate the autogenous healing capacity of concrete or engineer it [1], also through the incorporation of advanced functionalities, has been validated, in a much needed effort to assess their efficiency, through a multifold set of experimental tests and methodologies [2]. 
This paper will provide a synopsis on the topic. The importance will also be highlighted of the parameters to be experimentally identified, with the aim of "feeding" them as input to predictive modeling tools [3], which stand as the chain link between R\&D activity and the widespread adoption, also through code acceptance, of this technology into the construction market.

\section{Experimental Quantification of the Healing Capacity of Cement-Based Materials}

The quantification of the healing capacity of cement-based materials, be it based either on (stimulated) autogenous mechanisms or on an engineered process, involves a comprehensive set of activities. The range from creating a controlled "damage state" (a crack) in the material to the implementation of "healing conditions" suitably representing the intended functional and structural service scenario, to the evaluation of the sealing of the crack and of the recovery of the properties of interest, supported by the comprehension of the underlying mechanisms through the characterization of the healing products. As a matter of fact, the role of the experimental mechanics is crucial for the successful accomplishment of the aforementioned tasks.

The choice of the most appropriate experimental methodology to perform these tasks has to consider the property whose healing-related recovery has to be measured together with the fracture toughness of the investigated material which governs the stability of its post-cracking response and the whole cracking and healing evaluation procedure. Such a choice is the outcome of a complex decision making process which has to obey to a " $5 \mathrm{~W}$ " concept structured as follows:

"WHO": the material and its fracture toughness: under the denomination of cement-based construction materials a broad range of cementitious composites is nowadays numbered, which, starting from the quasi-brittle "conventional" concrete, has progressively evolved towards tougher and tougher ones, from ordinary Fiber Reinforced Concrete (FRC) to High Performance Fiber Reinforced Concrete and Cementitious Composites (HPFRC/HPFCRCs). These witness the progressive refinement of the "crack opening control concept", evolving up to a material composition designed through micromechanical concepts and based on the balance between fiber pullout and crack-tip toughness. This composition is able to provide, after the formation of the first crack, a through-crack stress redistribution of such extent that the formation of new multiple cracks is favored instead of the further opening of the previously formed one(s). The aforesaid mix composition, characterized by high binder content and low water-binder ratios, on the one hand makes it possible to achieve a strain hardening behavior characterized by stable multiple cracking before the onset of unstable crack localization. This results into the possibility of performing, in a stable manner and with a quite accurate control of the crack opening, tests such 4-point bending on un-notched prisms and even direct tension tests, which would be otherwise quite unstable in the case of plain concrete. On the other hand, the same composition is highly conducive to self-healing since, even after quite a long time, may leave significant amounts of binder un-hydrated and hence potentially available to undergo, together with carbonation, a delayed hydration reaction. This also makes it worth to tackle the quantification of self-healing through the recovery of mechanical properties. In fact, the postcracking strength at different levels of crack opening, are the parameters which have to be employed in the design of FRC/HPFRCC structures [4]. It is hence of the utmost importance not only to experimentally identify those parameters but also to assess, through likewise sound experimental methods, the possibility and extent of maintaining their "instantaneous" values along the structure service life. This calls into play the second " $\mathrm{W}$ ":

"WHAT": the requested performance recovery which has to be obtained thanks to combined effects of crack sealing and material healing, which could be either a durability or a mechanical one. In the first case the healing is evaluated by measuring a durability related parameter (e.g., sorptivity, water permeability, chloride ion penetration resistance) on the same specimen immediately after precracking and after the scheduled "curing time" under the selected exposure conditions. The durability property to be measured dictates the test methodology to be employed to measure the healing together with the methodology to be employed to create the crack to be healed. For example, chloride penetration tests generally employ cylinder specimens which could be pre-cracked by splitting tensile tests, though, in the case of plain concrete, such a test is quite unstable and producing 
a crack with a controlled width may be difficult. The same holds for water penetration tests. In case, fibers can help to stabilize the post-cracking response [5,6]. In the case of sorptivity, the crack through which the specimen water gain is triggered could be induced by means of 3-point bending on either notched or un-notched prisms, by performing the test in a crack-opening controlled mode through closed loop feedback systems. On the other hand, the evaluation of the healing through the recovery of mechanical properties is based on the comparative evaluation of the mechanical response of the same specimens to the same action, applied for pre-cracking the specimen first and after the scheduled healing time. Hence, it becomes of the utmost importance to test companion specimens which initially remain undamaged and undergo the same curing history as the cracked/healed ones and are then tested at the same age of the healing specimens. This only can allow to discriminate between healing-induced recovery and aging-induced evolution of the mechanical property of interest.

In view of this, the boundary conditions in which the healing recovery has to be evaluated ("WHERE" and "WHEN") are of paramount importance since they are meant to replicate at the lab-scale the structural service scenario under which the healing has to take place with reference to the intended application. The scenario encompasses the exposure conditions, with main reference to the (dis)continuous presence of water and/or moisture, temperature, pressure and possible presence of aggressive substances. Moreover, it has to consider the type and duration of actions which have caused cracking, and at which age, and which the structure may fully or partially continue to sustain along its service life. To the current state of knowledge, the foremost, though quite challenging, experimental need is the ability to replicate, as long as the healing goes, the presence of sustained and/or repeated through-crack stress states so to correctly reproduce their interaction with the healing phenomena and the long-term behavior of the material. The aforementioned through-crack stress states, which are implied by the intrinsic cohesive nature of concrete cracks as well as by the presence of conventional reinforcing bars or dispersed fiber reinforcement, may even not be tensile. Applications exist, such as in tunnel rings made of precast elements, where a crack formed at earlier ages in transient situations may later experience, in the persistent service design scenario, throughcrack compressive stresses, whose effect on the healing may be favorable. The interaction between the characteristics of the crack pattern, in terms of width and spacing, the exposure conditions and the nature and frequency of the sustained and/or repeated actions may also imply, in the case of R/C and FRC thanks to, e.g., improved bond, a stress redistribution capacity such that new freshly healable cracks may form in the non-crack-saturated free space between the existing cracks, instead of the progressively reopening of the same crack(s), which will obviously feature a lower and lower healing potential. In this respect it would also be advisable to study the effects of healing not only on the fundamental mechanical and durability related properties of the material but also on "mesoscale" structural mechanisms, such as the bond to reinforcement, which stands as a transfer to the structure scale of the healing-related performance recovery of the materials. An attempt in this direction has been made with reference to masonry structures by De Nardi et al. [7], who studied the effects of healing of lime mortars on the brick-mortar interface bond in structure subassemblies.

The complexity of the scenarios described above requires a likewise comprehensive characterization of the healing mechanisms [2], and hence the last but not least "W" "WHY", which can rely upon methods ranging from classical ones, such as Electronic Scanning Electron Microscope (SEM-ESEM), as well Thermo-Gravimetry Analysis (TGA), X-ray Diffraction and Fourier Transform Infrared (FTIR) Spectroscopy to less common ones, which may go even down to the visualization of the healing products through X-ray microtomography [8] and the characterization of the signature properties of the healing products through nano-indentation $[9,10]$.

As a matter of fact, the experimental characterization of the healing products has to be based not on a single test but rather on a thorough methodology combining visual crack sealing evaluation, measurement of durability related and/(or) mechanical recovery and establishment of mutual correlation between the garnered measurements, all completed by the micro- and, in case, nano-scale characterization of the healed cracks and healing products. 


\section{Definition of Healing-Related Indices of Performance Recovery}

Results garnered through the experimental methodologies summarized in the previous section need then to be "synthesized" into some kind of healing-related indices of performance recovery in order to, e.g., comparatively assess the efficiency of different healing techniques but with the aim of incorporating healing concept and beneficial outcomes into "to be formulated" design approaches. At a first instance, the quantification of crack-sealing through optical microscopy and image processing can lead to the definition of an Index of Crack Sealing (ICS), defined as:

$$
\text { ICS }=\frac{w_{\text {pre }} \text {-cracking }-w_{\text {post }} \text {-healing }}{w_{\text {pre }}-\text { cracking }}
$$

which will obviously depend on the multifold set of variables affecting and governing the healing mechanisms, and where $w$ denotes the crack width, as measured at the different stages of the experimental evaluation procedure. Commonly to all the other indices which will be hereafter reviewed, its values range between 0 (no-healing; $\mathrm{w}_{\text {post-healing }}=\mathrm{w}_{\text {pre-cracking) }}$ and 1 (complete healing; $\mathrm{W}_{\text {post-healing }}=0$ ).

It is worth here remarking that this index will only account for the closure visible at the surface of the crack, whereas internal reconstruction of the through crack material continuity, which will govern as well the recovery of the material performance can only be assessed by means of methods such as X-ray or neutron radiography and tomography, microcomputer-tomography scan and electrical methods [2]. These methods, though employed in a number of healing related studies, have hardly led so far to any quantification of crack sealing through index such as in Equation (1).

In the evaluation of self-healing by means of permeability tests, two main options have been proposed for the definition of an Index of Self-Healing (ISH). Equation (2), proposed in HealCON project [11], uses data from companion unhealed specimen as reference, while equation 3 , proposed by Roig-Flores et al. $[5,6]$ uses the same specimen and compares the water flow through it before and after healing:

$$
\begin{gathered}
\mathrm{ISH}_{\text {permeability }, 1}=\frac{\mathrm{w}_{\text {unhealed, } \mathrm{t}}-\mathrm{w}_{\text {healed }, \mathrm{t}}}{\mathrm{w}_{\text {unhealed, } \mathrm{t}}} \\
\mathrm{ISH}_{\text {permeability, } 2}=\frac{\mathrm{W}_{\text {initial }}-\mathrm{W}_{\text {final }}}{\mathrm{W}_{\text {initial }}}
\end{gathered}
$$

where $W_{\text {unhealed,t }}$ is the amount of water passing through the specimen's unhealed crack at time $t$, Whealed, is the amount of water passing through the specimen's healed crack at time $t, W_{\text {initial }}$ is the amount of water passing through the target specimen before healing and $\mathrm{W}_{\text {final, }}$ after healing. Gruyaert et al. [11] provided extensive explanation on the use of the aforementioned equations in the case of healing engineered through the use of superabsorbent polymers. Similarly, the following expression has been proposed to calculate a healing index from sorptivity tests:

$$
\mathrm{ISH}_{\text {sorptivity }}=\frac{\mathrm{S}_{\text {unhealed }}-\mathrm{S}_{\text {healed }}}{\mathrm{S}_{\text {unhealed }}-\mathrm{S}_{\text {uncracked }}}
$$

where Sunhealed, Shealed and Suncracked are the sorptivity indices for the cracked and unhealed specimen, for the cracked and healed specimen and for the uncracked reference specimen respectively.

The definition of healing indices representative of the recovery of mechanical properties requires comparing the pre-cracking response of the material with the post-healing one, as measured on the same specimen, e.g., in terms of nominal stress vs. crack opening.

An Index of Strength Recovery (ISR) can be defined, with reference to notation shown in Figure 1, as:

$$
\mathrm{ISR}=\frac{\mathrm{f}_{\text {peak,post }} \text {-conditioning }-\sigma_{\text {unloading,pre-cracking }}}{\mathrm{f}_{\text {peak,pre-cracking }}-\sigma_{\text {unloading,pre-cracking }}}
$$

which fits for plain concrete, ordinary FRC and strain softening HPFRCC, whereas for strain/deflection hardening HPFRCC, if pre-cracked in the pre-peak regime, different expressions have to be considered, in order to distinguish the effects of healing from the pre-peak hardening inborn in the material response. Indices of ductility recovery have also been proposed [12,13]. 


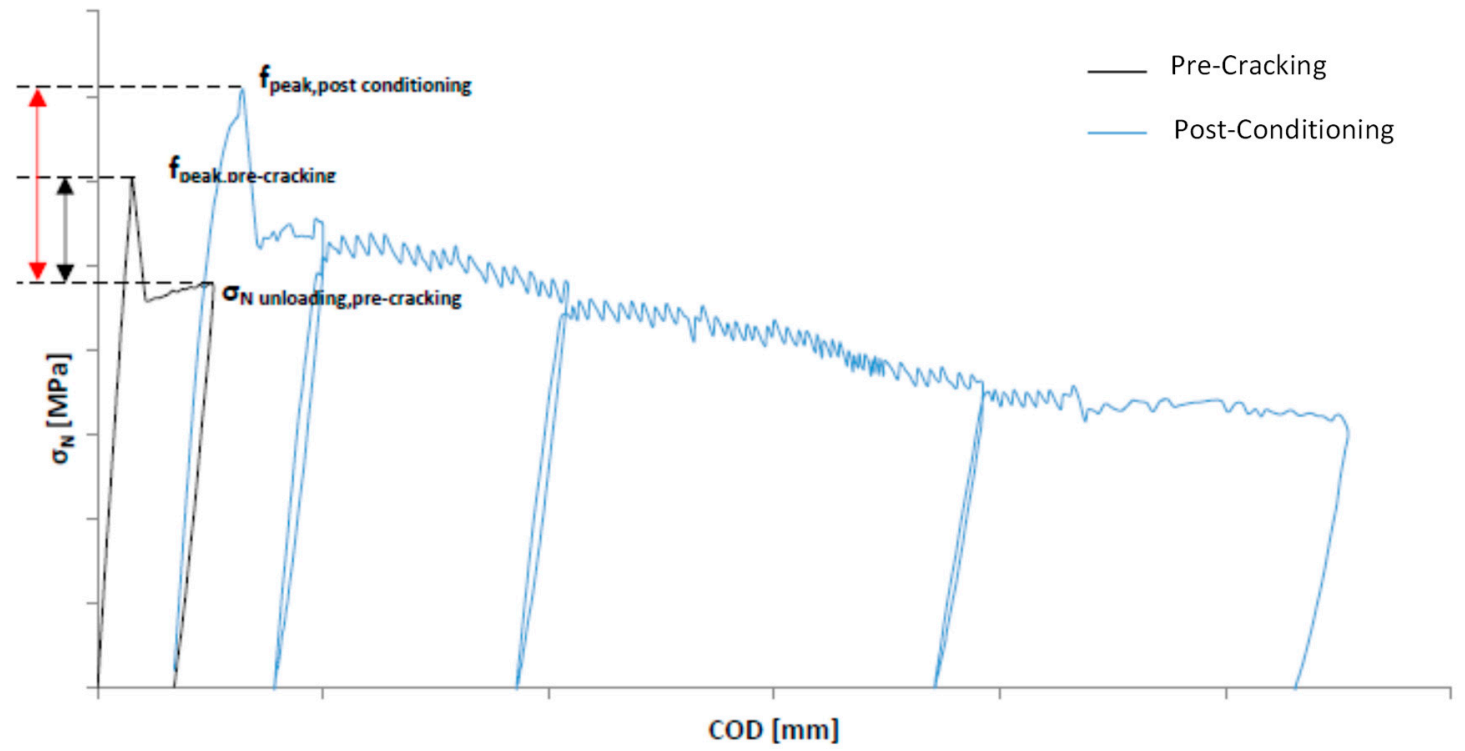

(a)

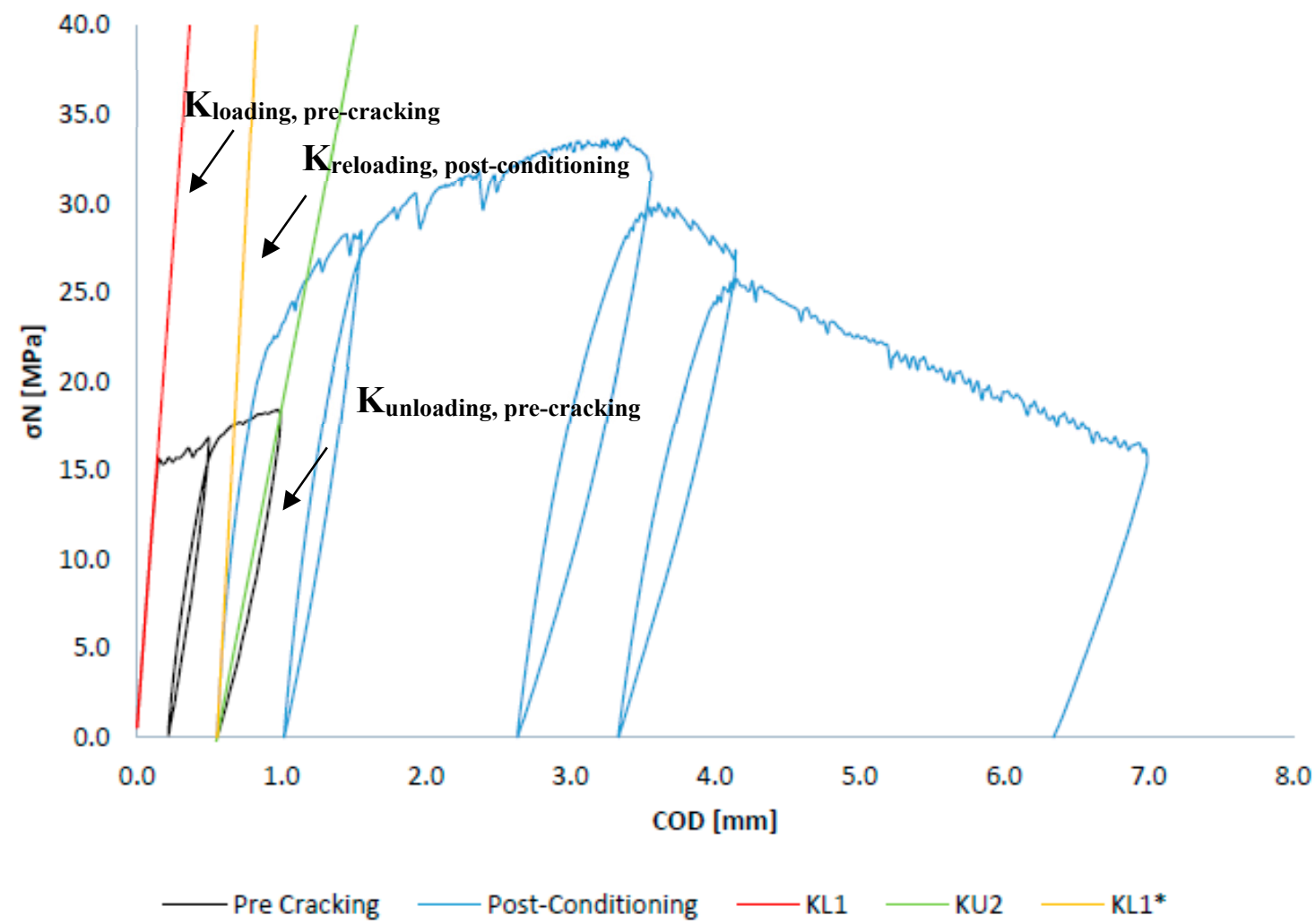

(b)

Figure 1. Notation for the definition of Index of Strength Recovery (a) and of Damage Recovery (b) [12].

Similarly, the comparison of the pre-cracking vs. post-healing performance in terms of stiffness can lead to the definition of an Index of Damage Recovery (IDR - see notation in Figure 1):

$$
\mathrm{IDR}=\frac{\mathrm{K}_{\text {loading,post-conditioning }}-\mathrm{K}_{\text {unloading,pre-cracking }}}{\mathrm{K}_{\text {loading,pre-cracking }}-\mathrm{K}_{\mathrm{unloading}} \text { pre-cracking }}
$$

Ferrara et al. $[12,13]$ have compared the strength and damage recovery, as from Equations (5) and (6) to the index of crack sealing, as from Equation (1). They observed that a "surface crack sealing" of at least $60 \%$ is necessary before a significant mechanical healing can be observed. As a 
matter of fact, a higher surface crack sealing, which is reflected in the Index of Crack Sealing, likely corresponds to a more complete internal crack sealing, thus justifying the results.

Comparing the pre-cracking and post-conditioning nominal bending stress vs. crack opening displacement (COD) curves a method to indirectly estimate the crack sealing was proposed [14]. It consists in operating a "backward" shifting along the COD axis, of the stress-COD post-conditioning curve, until the curve measured in the pre-cracking test on the virgin undamaged sample is met. The new value of the "origin" COD can be estimated by drawing, from the aforementioned point on the pre-cracking curve, an unloading branch parallel to the last unloading previously measured on the virgin sample itself (Figure 2). Moreover, by performing, during the pre-cracking and postconditioning tests, repeated loading-unloading cycles (Figure 1b), the evolution of damage (stiffness decay) as a function of the crack opening could be estimated, and damage vs. crack opening curves could be built. By identifying the points representative of damage-COD state in the post-conditioning test and operating a "backward shifting" along the COD axis until the fitted damage-COD evolution curve of the virgin specimen is met, the amount of this backward shifting can be assumed as an indicator of the crack closure effect produced by the self-healing phenomena (Figure 2).

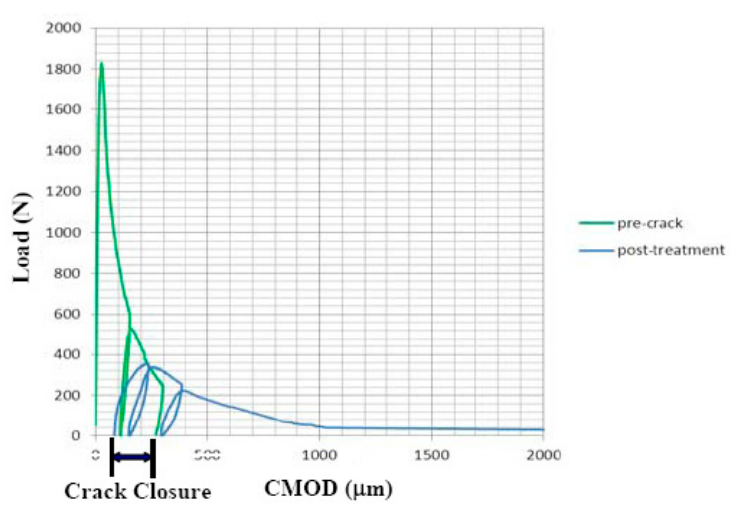

(a)

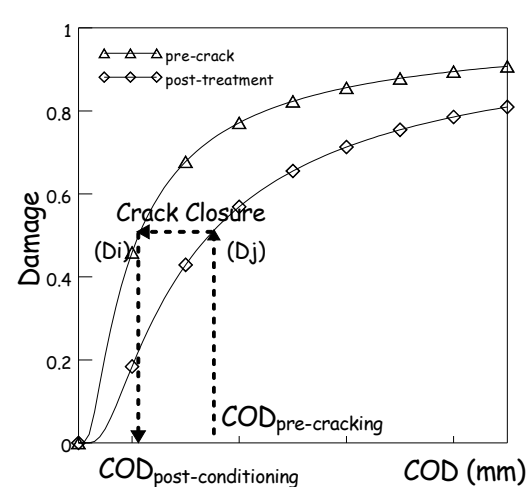

(b)

Figure 2. Estimation of crack closure from nominal stress (a) and damage (b) vs. COD curves [13,14].

\section{Conclusions}

In this paper the fundamental concepts which have to govern the choice of the experimental quantification of the self-sealing capacity of cement-based materials have been discussed, also providing examples of such a quantification through the definition of healing indices. This step has to serve as input for predictive models, including Life Cycle Analysis, which only can provide the necessary extension to the engineering application scale needed to incorporate self-healing concepts and outcomes into durability based design approaches and foster their widespread market penetration into the construction market and structure and infrastructure engineering world.

Author Contributions: This paper is based on discussions and collaboration among the authors in the framework of the COST Action acknowledged below. All the authors equally contributed to the paper.

Acknowledgments: The authors acknowledge the support from EU COST Action CA 15202 "SARCOS".

Conflicts of Interest: The authors declare no conflict of interest.

\section{References}

1. De Belie, N.; Gruyaert, E.; Al-Tabbaa, A.; Antonaci, P.; Baera, C.; Bajare, D.; Darquennes, A.; Davies, R.; Ferrara, L.; Jefferson, T.; et al. A review of self-healing concrete for damage management of structures. Adv. Mater. Interfaces 2018, doi:10.1002/admi.201800074.

2. Ferrara, L.; Van Mullem, T.; Alonso, M.C.; Antonaci, P.; Borg, R.P.; Cuenca, E.; Jefferson, A.; Ng, P.L.; Peled, A.; Roig, M.; et al. Experimental characterization of the self-healing capacity of cement based materials and its effects on the material performance: A state of the art report by COST Action SARCOS WG2. Constr. Build. Mater. 2018, 167, 115-142. 
3. Jefferson, T.; Javierre, E.; Lee Freeman, B.; Zaoui, A.; Koenders, E.; Ferrara, L. Research progress on numerical models for self-healing cementitious materials. Adv. Mater. Interfaces 2018, doi:10.1002/admi.201701378.

4. Taerwe, L.; Matthys, S. Fib Model Code 2010 for Concrete Structures; Ernst \& Sohn: Berlin, Germany, 2013; Volume 2.

5. Roig-Flores, M.; Moscato, S.; Serna, P.; Ferrara, L. Self-healing capability of concrete with crystalline admixtures in different environments. Constr. Build. Mater. 2015, 86, 1-11.

6. Roig Flores, M.; Pirritano, F.; Serna Ros, P.; Ferrara, L. Effect of crystalline admixtures on the self-healing capability of early-age concrete studied by means of permeability and crack closing tests. Constr. Build. Mater. 2016, 114, 447-457.

7. De Nardi, C.; Cecchi, A.; Ferrara, L. The influence of self-healing capacity of lime mortars on the behavior of brick-mortar masonry subassemblies. In Proceedings of the MURICO-5, Bologna, Italy, 28-30 June 2017. Also in Key Eng. Mater. 2017, 747, 465-471.

8. Snoeck, D.; Dewanckele, J.; Cnudde, V.; De Belie, N. X-ray computed microtomography to study autogenous healing of cementitious materials promoted by superabsorbent polymers. Cem. Concr. Compos. 2016, 65, 83-93.

9. $\mathrm{Xu}, \mathrm{J} . ; \mathrm{Yao}, \mathrm{W}$. Multiscale mechanical quantification of self-healing concrete incorporating non-ureolytic bacteria-based healing agent. Cem. Concr. Res. 2014, 64, 1-10.

10. Ferrara, L.; Kassavetis, S.; Lo Monte, F.; Stefanidou, M. Characterization of self-healing reaction products in cementitious mortars via nano-indentation: A cross-collaboration in the framework of COST Action SARCOS. In Proceedings of the NICOM 6, Hong Kong, China, 2-5 December 2018.

11. Gruyaert, E.; Debbaut, D.; Snoeck, D.; Díaz, P.; Arizo, A.; Tziviloglou, E.; Schlangen, E.; De Belie, N. Selfhealing mortar with $\mathrm{pH}$-sensitive superabsorbent polymers: Testing of the sealing efficiency by water flow tests. Smart Mater. Struct. 2016, 25, 084007.

12. Ferrara, L.; Krelani, V.; Moretti, F. Autogenous healing on the recovery of mechanical performance of HPFRCCs: Part 2-Correlation between healing of mechanical performance and crack sealing. Cem. Concr. Compos. 2016, 73, 299-315.

13. Ferrara, L.; Krelani, V.; Moretti, F.; Roig-Flores, M.; Serna, P. Effects of autogenous healing on the recovery of mechanical performance of HPFRCCs: Part 1. Cem. Concr. Compos. 2017, 83, 76-100.

14. Ferrara, L.; Krelani, V.; Carsana, M. A fracture testing based approach to assess crack healing of concrete with and without crystalline admixtures. Constr. Build. Mater. 2014, 68, 515-531.

(C) 2018 by the authors. Licensee MDPI, Basel, Switzerland. This article is an open access article distributed under the terms and conditions of the Creative Commons Attribution (CC BY) license (http://creativecommons.org/licenses/by/4.0/). 